\title{
Increased alpha-synuclein tear fluid levels in patients with Parkinson's disease
}

\author{
Fabian Maass $\circledast^{1 凶}$, Sebastian Rikker ${ }^{1}$, Vivian Dambeck ${ }^{1,5,6}$, Carmina Warth $^{1,6}$, \\ Lars Tatenhorst ${ }^{1,3,5,6}$, Ilona Csoti ${ }^{2}$, Matthias Schmitz ${ }^{1,3}$, Inga Zerr ${ }^{1,3}$, Andreas Leha ${ }^{4}$, \\ Mathias Bähr ${ }^{1,5}$ \& Paul Lingor $\mathbb{B}^{1,3,5,6,7}$
}

The objective of the study was to estimate if altered levels of alpha-synuclein can be detected in tear fluid of patients with Parkinson's disease (PD). Therefore, tear fluid samples of 75 PD patients, 75 control subjects and 31 atypical Parkinsonian patients were collected and analyzed in triplicates using an ultra-sensitive single molecule array (SIMOA) system and applying a human alpha-synuclein immunoassay. In PD, levels of total soluble alpha-synuclein were significantly increased compared to control subjects ( $p=0.03$; AUC PD vs. controls 0.60 ). There was no difference comparing PD patients stratified by Hoehn \& Yahr stages and atypical Parkinsonian syndromes stratified by tauopathies and non-PD-synucleinopathies against each other $(p>0.05)$. In conclusion, alpha-synuclein can be detected and quantified in tear fluid, revealing small but significant differences in total alpha-synuclein levels between PD and control subjects. Tear fluid can be collected non-invasively and risk-free, therefore presenting a promising source for further biomarker research.

Alpha-synuclein (aSyn) is an essential component of the Lewy body and represents the hallmark protein of Parkinson's disease (PD) pathology ${ }^{1}$. Consequently, the potential of aSyn to function as a biomarker for PD was analyzed in multiple different biofluids (e.g. serum and CSF). However, high intra- and interstudy variability in aSyn levels and the lack of discriminatory power prevent its use as individual biomarker for diagnosis of $\mathrm{PD}^{2}$.

Previously, aSyn positivity in PD was demonstrated in the salivary gland $s^{3}$, but saliva itself did not yield a clear difference in aSyn levels between PD and controls ${ }^{4}$, also due to preanalytical difficulties (e.g. blood contamination, bioactive enzymes ${ }^{5}$ ).

The lacrimal gland and the salivary gland share the same parasympathetic innervation originating in the brainstem yielding a common connection for the transmission of aSyn pathology (Fig. 1a). Tear fluid (TF) is a cost efficient, easily and non-invasively collectable body fluid. Its analysis thus presents a promising alternative approach considering its superior preanalytical characteristics.

Recently, a multiplex ELISA was used to quantify aSyn levels in $\mathrm{TF}^{6}$. However, conventional ELISA-based analyses have yielded inconsistent results on aSyn biofluid levels in the past (e.g. conflicting aSyn plasma levels ${ }^{7}$ ) whereas the application of ultrasensitive single molecule array (SIMOA) might yield more sensitive and reliable quantification results, as recently described for the detection of aSyn plasma levels in $\mathrm{PD}^{8}$.

Here we present the first study of aSyn quantification in TF using SIMOA in a comprehensive cohort of PD patients, control subjects and atypical Parkinsonian syndromes.

\footnotetext{
${ }^{1}$ Department of Neurology, University Medical Center, Göttingen, Germany. ${ }^{2}$ Gertrudis Clinic Parkinson-Center, Biskirchen, Germany. ${ }^{3} \mathrm{DZNE}$, German Center for Neurodegenerative Diseases, Munich and Göttingen, Germany. ${ }^{4}$ Department of Medical Statistics, University Medical Center, Göttingen, Germany. ${ }^{5}$ Cluster of Excellence Nanoscale Microscopy and Molecular Physiology of the Brain (CNMPB), Göttingen, Germany. ${ }^{6}$ Center for Biostructural Imaging of Neurodegeneration (BIN), University Medical Center, Göttingen, Germany. ${ }^{7}$ Technical University of Munich, School of Medicine, Klinikum rechts der Isar, Department of Neurology, 81675, Munich, Germany. ${ }^{\bowtie e}$-mail: fabian. maass@med.uni-goettingen.de
} 
a
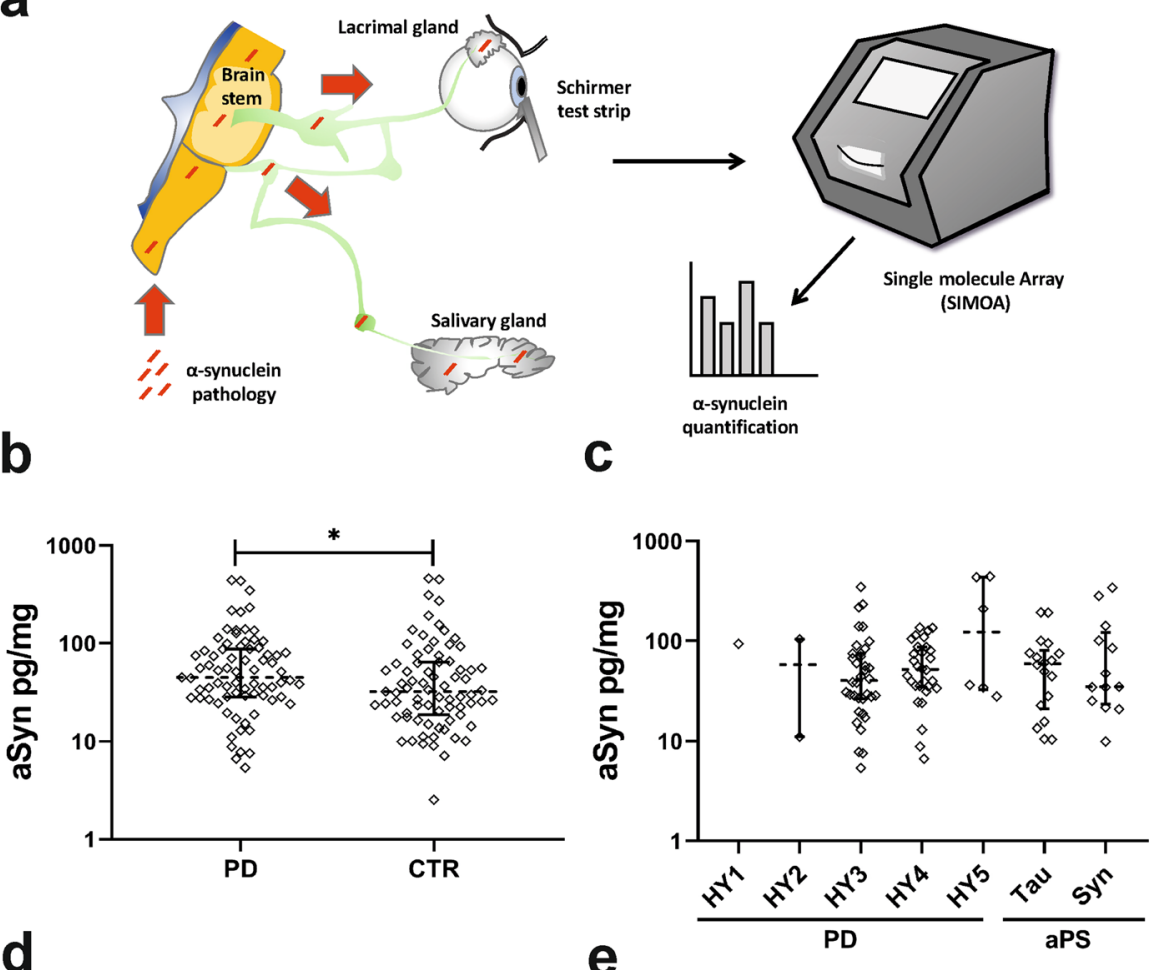

d

e

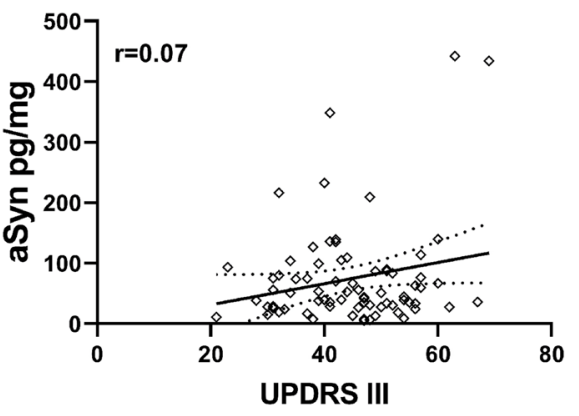

Figure 1. (a) The lacrimal gland and the salivary gland share the same parasympathetic innervation originating in the brainstem yielding a common connection for the transmission of aSyn pathology. Tear fluid was collected using Schirmer strips. aSyn levels were quantified applying single molecule array (SIMOA) (b) aSyn tear fluid levels in PD/CTR $(\mathrm{p}=0.03)$. (c) aSyn levels in PD stratified by Hoehn \& Yahr stage (HY) and in atypical Parkinsonian (aPS) syndromes stratified by tauopathies (Tau) and synucleinopathies (Syn). (d) ROC curve for the discrimination of PD and CTR. (e) Correlation between aSyn and UPDRS III. Data in (b,c) is presented as single values with median and interquartile range on a $\log 10$ scaled $y$-axis. $\mathrm{PD}=$ Parkinson's disease, $\mathrm{CTR}=$ control subjects.

\section{Results}

150 participants (PD $n=75$, CTR $n=75$ ) were enrolled for primary analysis. There was no significant difference in gender, age or ophthalmological comorbidities between PD and CTR ( $p>0.05$, summarized in Table 1). Additionally, 18 patients with tauopathies (PSP $n=13, C B S n=5$ ) and 13 patients with other synucleinopathies (MSA-C $\mathrm{n}=2$, MSA-P $\mathrm{n}=11$ ) were included for secondary analysis.

There was neither a significant difference in TF total protein concentrations $(2.10 \mu \mathrm{g} / \mu \mathrm{l}$, IQR $1.65-2.86 \mathrm{vs}$. $2.07 \mu \mathrm{g} / \mu \mathrm{l}, \mathrm{IQR} 1.31-2.75 ; \mathrm{p}=0.64)$ nor in sum wetting length $(15 \mathrm{~mm}$, IQR $8-24$ vs. $15 \mathrm{~mm}, \mathrm{IQR} 8-28 ; \mathrm{p}=0.55)$ comparing PD and CTR.

In PD, levels of aSyn were significantly increased compared to CTR (44.91 pg/mg, IQR 28.26-87.16 vs. $32.02 \mathrm{pg} / \mathrm{mg}$, IQR 18.57-64.51; $\mathrm{p}=0.03$; mean triplicate CV 7.9\%; Fig. 1b), resulting in a moderate discriminative value with an AUC of 0.60 ( $\mathrm{p}=0.03,95 \%$ CI $0.51-0.69$, sensitivity $0.44 /$ specificity 0.80 on Youden index; Fig. $1 \mathrm{~d})$.

There was no difference in aSyn levels comparing PD patients stratified by H\&Y stages and atypical Parkinsonian syndromes stratified by tauopathies and non-PD-synucleinopathies against each other (H\&Y1 93.54 pg/mg, IQR NA; H\&Y2 57.58 pg/mg, IQR 11.09-104.1; H\&Y3 40.21 pg/mg, IQR 26.35-75.19; H\&Y4 $51.86 \mathrm{pg} / \mathrm{mg}$, IQR 34.63-86.89; H\&Y5 $122.8 \mathrm{pg} / \mathrm{mg}$, IQR 32.55-436.3; tauopathies 59.12 pg/mg, IQR 20.83-80.35; synucleinopathies $34.78 \mathrm{pg} / \mathrm{mg}$, IQR $23.31-121.2 ; \mathrm{p}=0.73$; Fig. 1c). 


\begin{tabular}{|l|l|l|l|l|l|}
\hline & PD & CTR & P-value $^{*}$ & Tauo-pathies* & $\begin{array}{l}\text { Non-PD Synucleino- } \\
\text { pathies }\end{array}$ \\
\hline Patients $(n)$ & 75 & 75 & & 18 & 13 \\
\hline Age $($ years $)$ & $70(64-76)$ & $70(62-79)$ & 0.77 & $71(67-77)$ & $71(66-78)$ \\
\hline Male/female (\% female) & $49 / 26(34.7 \%)$ & $50 / 25(33.3 \%)$ & 0.86 & $10 / 8(44.4 \%)$ & $8 / 5(38.5 \%)$ \\
\hline H\&Y stage & $3.0(3.0-4.0)$ & NA & & $4.0(3.4-4.0)$ & $3.0(3.0-4.0)$ \\
\hline Disease duration (years) & $7.0(4.0-10.0)$ & NA & & $2.5(2.0-4.0)$ & $4.0(2.0-6.0)$ \\
\hline UPDRS III & $45(38-52)$ & NA & & $39(31-52)$ & $36(27-54)$ \\
\hline Glaucoma $n(\%)$ & $1(1.3 \%)$ & $4(5.3 \%)$ & 0.17 & $0(0.0 \%)$ & $0(0.0 \%)$ \\
\hline Macular degeneration $(n)(\%)$ & $1(1.3 \%)$ & $1(1.3 \%)$ & 1.00 & $1(5.6 \%)$ & $0(0.0 \%)$ \\
\hline Cataract $(n)(\%)$ & $9(12.0 \%)$ & $7(9.3 \%)$ & 0.60 & $1(5.6 \%)$ & $0(0.0 \%)$ \\
\hline Any other eye disease $(n)(\%)$ & $6(8.0 \%)$ & $7(9.3 \%)$ & 0.77 & $1(5.6 \%)$ & $1(7.7 \%)$ \\
\hline Contact lenses $n(\%)$ & $3(4.0 \%)$ & $1(1.3 \%)$ & 0.31 & $0(0.0 \%)$ & $0(0.0 \%)$ \\
\hline Medical eye drops $n(\%)$ & $5(6.7 \%)$ & $6(8.0 \%)$ & 0.75 & $2(11.1 \%)$ & $0(0.0 \%)$ \\
\hline Lubricants $(n)(\%)$ & $10(13.3 \%)$ & $4(5.3 \%)$ & 0.09 & $2(11.1 \%)$ & $0(0.0 \%)$ \\
\hline
\end{tabular}

Table 1. Characteristics of the study population. Data is presented as median $\left(25^{\text {th }}-75^{\text {th }}\right.$ percentile $)$. $\mathrm{PD}=$ Parkinson's disease, $\mathrm{CTR}=$ control subjects, $\mathrm{NA}=$ not applicable. UPDRS $=$ Unified Parkinson's Disease Rating Scale. ${ }^{\text {P }}$-values reported for the comparison of PD and CTR. *Tauopathies including PSP $n=13$, CBS $\mathrm{n}=5$. ${ }^{\mathrm{S}}$ Synucleinopathies including MSA-C $\mathrm{n}=2, \mathrm{MSA}-\mathrm{P} \mathrm{n}=11$.

There was a weak correlation between age and aSyn levels in CTR $(r=0.28 ; \mathrm{p}=0.02)$ but not in PD $(r=0.19$; $\mathrm{p}=0.10)$. aSyn levels were not significantly correlated with disease duration or UPDRS III score $(\mathrm{r}=0.05$, $\mathrm{p}=0.70 ; \mathrm{r}=0.07, \mathrm{p}=0.57$, Fig. 1e).

A binary logistic regression model was fitted to distinguish PD from CTR based on aSyn levels and potentially confounding factors age, gender, application of medical eye drops or lubricants, presence of eye diseases or usage of contact lenses. This model still yielded a significant difference in aSyn between PD and CTR $(\mathrm{p}=0.048)$ without significant confounding effects by the mentioned factors.

\section{Discussion}

In a proof-of-principle approach, we demonstrate that aSyn can be detected and quantified in TF by ultra-sensitive SIMOA, revealing increased values of total soluble aSyn in PD compared to control subjects. Quantification was performed in multiple measurements (triplicates) per subject, yielding a mean triplicate CV of $7.9 \%$.

The performance of SIMOA for aSyn detection in tear fluid should be validated in further trials and currently, there is no gold standard available which could be referred to. Previous studies reporting aSyn concentrations in other biofluids used different in-house developed or commercially available ELISA systems ${ }^{2,9}$. Here, we applied SIMOA instead of conventional ELISA due to its known capability for ultrasensitive protein measurements and increasing evidence for superior detection characteristics in protein quantification, e.g. for the quantification of neurofilaments ${ }^{10,11}$.

For the current study, we preferred the application of Schirmer strips for collection and analysis of reflex tears instead of the usage of microcapillary tubes or anesthesia for the collection of basal tears. Using Schirmer strips is common in clinical practice and has many advantages compared to capillary tube sampling ${ }^{12}$. It is very safe and well tolerated by the patients, therefore it can be easily translated into clinical routine. Avoiding anesthesia additionally allows to exclude bias due to potential influences by the medication.

The source of TF aSyn is uncertain and a release into TF by innervating nerve structures in the course of aSyn transmission might be involved. Being an ultrafiltrate of plasma ${ }^{13}$, TF aSyn might also be blood derived. Ng and colleagues recently applied SIMOA for the detection of total soluble aSyn in plasma samples showing results similar to our TF data, revealing slightly increased aSyn values in PD and a distinct overlap with the control group, respectively ${ }^{8}$.

Only a few studies have analyzed TF in PD and recently aSyn was shown to be decreased in TF of PD patients ${ }^{6}$. In this study, however, anesthetic eye drops were instilled prior to performance of the Schirmer test and aSyn levels were quantified using an ELISA instead of an ultra-sensitive SIMOA.

We further report that aSyn can be detected in TF of patients with atypical Parkinsonian syndromes. Our analysis of an exploratory cohort showed no clear difference in aSyn values between tauopathies (PSP, CBD), non-PD-synucleinopathies (MSA-P, MSA-C) and PD. Here, a larger number of these less frequently occurring patients needs to be analyzed in order to draw robust conclusions. Nevertheless, we demonstrate that the measurements in both groups of atypical Parkinsonian patients are in a similar range compared to PD patients.

We detected no significant differences in aSyn values in PD patients stratified for H\&Y stages, which is comparable to the results that were reported for aSyn plasma levels quantified by SIMOA ${ }^{8}$. However, most patients were classified H\&Y 3 or 4, and we thus cannot exclude that patients in other stages could show more pronounced changes. Likewise, there was also no significant correlation between aSyn levels and UPDRS III motor score.

With its moderate discriminative value (AUC 0.60), total soluble TF aSyn will not be sufficient as an individual biomarker for diagnosis, but the value of easily collectable TF remains promising. Further validation in independent and larger cohorts including patients with other disease entities is needed to confirm our results. 
So far, only one further group recently reported total aSyn quantification in tear fluid, applying a Luminex ELISA in contrast to our SIMOA approach ${ }^{6}$. Both approaches allow for alpha-synuclein detection, yielding evidence for the occurrence of aSyn in TF accompanied by small differences in total aSyn levels between PD and control subjects (AUC 0.63 and AUC 0.60, respectively). Distinct preanalytical and analytical differences prevent direct comparison of both studies (application of anesthetic eye drops, different types of antibodies, different detection and quantificationmethods). Taking together the data from both studies, it can be assumed that total aSyn will not be sufficient as a diagnostic biomarker for the discrimination of PD patients and controls. However, aSyn could be part of a biomarker panel and its reliable detection in an easily accessible biofluid, such as tear fluid, is therefore of particular interest ${ }^{14}$.

As we could recently show, TF can also be analyzed by proteomics and PD patients show a differential protein composition as compared to controls ${ }^{15}$. Therefore, protein combinations could potentially act as biomarkers rather than individual proteins. In addition, the analysis of aggregated aSyn isoforms using protein-misfolding cyclic amplification (PMCA) or real-time quaking-induced conversion (RT-QuIC) ${ }^{16}$ could complement SIMOA to improve discriminative power for the translation of an individual biomarker into clinical routine.

\section{Methods}

Participants. PD patients diagnosed according to MDS criteria ${ }^{17}$ and control subjects (CTR) without clinical signs of neurodegeneration but comparable age- and gender characteristics were consecutively enrolled at the out- and in-patient clinics of the Department of Neurology of the University Medical Center Göttingen and at the Gertrudis Parkinson Center Biskirchen, Germany between August 2018 and May 2019 for primary analysis. All patients were included independent of disease severity. Patients with Progressive Supranuclear Palsy (PSP), Corticobasal Syndrome (CBS) and Multiple System Atrophy (MSA-P, MSA-C) according to acknowledged criteria ${ }^{18-20}$ were also enrolled in this period and additionally selected from the biobank of the Department of Neurology, Göttingen based on availability of TF samples. Patients underwent neurological examination and history taking by movement disorder specialists. Ophthalmological comorbidities and topical eye medication were recorded. UPDRS score part III was applied for assessment of motor function.

Ethical approval and informed consent. A permission of the local ethics committee has been obtained prior to the initiation of the study (Ethics Committee of the University Medical Center Göttingen Nr.: 13/11/12; 10/8/18). All participants voluntarily participated in the study. Informed consent for study participation was obtained from all patients and control subjects. The study conforms with the Code of Ethics of the World Medical Association (Declaration of Helsinki).

Sample collection and preparation. TF samples were collected from both eyes using Schirmer test strips (Optitech, Allahabad, India). Strips were placed at the inferior eye lid margin for $8 \mathrm{~min}$ and wetting length was noted, respectively. No topical anesthesia was used for sample collection. Samples were immediately frozen after collection and stored in polypropylene tubes at $-80^{\circ} \mathrm{C}$ until further analysis.

TF protein was eluted by adding RIPA buffer (Thermo Fisher Scientific, Waltham, MA) with protease and phosphatase inhibitor (cOmplete and PhosStOP; Roche, Basel, Switzerland) with subsequent centrifugation at $16000 \mathrm{~g}$ for $30 \mathrm{~min}$. Samples of both sides were pooled for analysis. Total protein content was determined using a bicinchoninic acid assay (Thermo Fisher Scientific).

Total soluble aSyn was quantified using an ultra-sensitive single molecule array detection system (SR-X, Quanterix, Billerica, MA) applying a human aSyn immunoassay (Discovery Kit 102233, Quanterix; details are given in the supplement). A positive aSyn control ( $1 / 3$ concentration of manufacturer's LOQ or lowest calibrator) was additionally added as duplicate on each plate to confirm that potential values between default LOQ $(4.12 \mathrm{pg} / \mathrm{ml}$, defined by the manufacturer applying a coefficient of variability $(\mathrm{CV})<20 \%$ with $80-120 \%$ recovery, mean recovery $101.1 \%$ ) and $1 / 3$ of default LOQ $(1.373 \mathrm{pg} / \mathrm{ml}$; calculated mean intra-assay CV $13.8 \%$, mean inter-assay CV $19.6 \%$, recovery $68-124 \%$, mean recovery $99 \%$ ) are still sufficient for quantification and further statistical analysis. All measurements were performed in triplicates using a 1:10 dilution. Results were normalized to total TF protein concentrations.

Statistical analysis. Quantitative data were compared using Mann-Whitney test. Qualitative data were compared using Chi-Squared test. 95\% confidence interval (CI) of the area under the ROC curve (AUC) was calculated according Wilson/Brown method. One-way ANOVA on ranks was applied to compare PD stratified by Hoehn and Yahr (H\&Y) and atypical Parkinsonian patients. A binary logistic regression model was fitted to $\log 10$-transformed data to control for potential confounders. Correlation between two variables was quantified using spearman's rho. Due to the lack of pre-existing data on TF aSyn quantification at the time of study initiation, valid sample size predetermination was not applicable. All analyses were performed using GraphPad Prism 8.0.1. or the R-language 3.5.3 with corresponding packages.

\section{Data availability}

The datasets analyzed during the current study are available from the corresponding author on reasonable request.

Received: 7 January 2020; Accepted: 5 May 2020;

Published online: 22 May 2020

\section{References}

1. Spillantini, M. G. et al. $\alpha$-Synuclein in Lewy bodies. Nature 388, 839 (1997)

2. Eusebi, P. et al. Diagnostic utility of cerebrospinal fluid $\alpha$-synuclein in Parkinson's disease: A systematic review and meta-analysis. Mov. Disord. 32, 1389-1400 (2017). 
3. Schneider, S. A. et al. Can we use peripheral tissue biopsies to diagnose Parkinson's disease? A review of the literature. Eur. J. Neurol. 23, 247-261 (2016).

4. Goldman, J. G. et al. Cerebrospinal fluid, plasma, and saliva in the BioFIND study: Relationships among biomarkers and Parkinson's disease Features. Mov. Disord. 33, 282-288 (2018).

5. Vivacqua, G., Mancinelli, R., Belvisi, D., Suppa, A. \& Berardelli, A. Detection of $\alpha$-Synuclein in Saliva: The Importance of Preanalytical Assessment. Mov. Disord. 33, 1029-1030 (2018).

6. Hamm-Alvarez, S. F. et al. Oligomeric $\alpha$-synuclein is increased in basal tears of Parkinson's patients. Biomark. Med. bmm-2019-0167 https://doi.org/10.2217/bmm-2019-0167 (2019).

7. Bougea, A. et al. Plasma alpha-synuclein levels in patients with Parkinson's disease: a systematic review and meta-analysis. Neurol. Sci. 40, 929-938 (2019).

8. Ng, A. S. L. et al. Plasma alpha-synuclein detected by single molecule array is increased in PD. Ann. Clin. Transl. Neurol. 6, 615-619 (2019).

9. Parnetti, L., Cicognola, C., Eusebi, P. \& Chiasserini, D. Value of cerebrospinal fluid $\alpha$-synuclein species as biomarker in Parkinson's diagnosis and prognosis. Biomarkers in Medicine https://doi.org/10.2217/bmm.15.107 (2016).

10. Rissin, D. M. et al. Single-molecule enzyme-linked immunosorbent assay detects serum proteins at subfemtomolar concentrations. Nat. Biotechnol. https://doi.org/10.1038/nbt.1641 (2010).

11. Verde, F. et al. Neurofilament light chain in serum for the diagnosis of amyotrophic lateral sclerosis. J. Neurol. Neurosurg. Psychiatry 90, 157-164 (2019).

12. Posa, A. et al. Schirmer strip vs. capillary tube method: Non-invasive methods of obtaining proteins from tear fluid. Ann. Anat. https://doi.org/10.1016/j.aanat.2012.10.001 (2013).

13. Mircheff, A. K. Lacrimal fluid and electrolyte secretion: a review. Curr. Eye Res. 8, 607-17 (1989).

14. Hall, S. et al. Accuracy of a panel of 5 cerebrospinal fluid biomarkers in the differential diagnosis of patients with dementia and/or parkinsonian disorders. Arch. Neurol. 69, 1445-1452 (2012).

15. Boerger, M. et al. Proteomic analysis of tear fluid reveals disease-specific patterns in patients with Parkinson's disease - A pilot study. Parkinsonism Relat. Disord. https://doi.org/10.1016/j.parkreldis.2019.03.001 (2019).

16. Paciotti, S., Bellomo, G., Gatticchi, L. \& Parnetti, L. Are We Ready for Detecting $\alpha$-Synuclein Prone to Aggregation in Patients? The Case of \&quot;Protein-Misfolding Cyclic Amplification\&quot; and \&quot;Real-Time Quaking-Induced Conversion\&quot; as Diagnostic Tools. Front. Neurol. 9, 415 (2018).

17. Postuma, R. B. et al. MDS clinical diagnostic criteria for Parkinson’s disease. Mov. Disord. 30, 1591-1601 (2015).

18. Litvan, I. et al. Clinical research criteria for the diagnosis of progressive supranuclear palsy (Steele-Richardson-Olszewski syndrome): report of the NINDS-SPSP international workshop. Neurology 47, 1-9 (1996).

19. Armstrong, M. J. et al. Criteria for the diagnosis of corticobasal degeneration. Neurology 80, 496-503 (2013).

20. Gilman, S. et al. Second consensus statement on the diagnosis of multiple system atrophy. Neurology 71, 670-6 (2008).

\section{Acknowledgements}

The authors gratefully appreciate the participation of our patients in this study. We thank our Parkinson's and study nurses Gudrun Leyerer and Jennifer Heinemann for their excellent assistance. We also thank Natalia Koleva-Alazeh for her assistance in patient recruitment and Peter Lange for providing helpful advice on assayrelated issues. This study was funded by: I2A Grant of the DZNE (P.L.); DFG Research Center for Nanoscale Microscopy and Molecular Physiology of the Brain (CNMPB) (V.D., L.T., P.L., M.B.); Forschungskolleg TRANSMED Göttingen, which was supported by the Ministerium für Wissenschaft und Kultur, Niedersachsen, Germany (F.M.). We acknowledge support by the Open Access Publication Funds of the Göttingen University.

\section{Author contributions}

Design, conceptualization and execution of the study: F.M., S.R., V.D., C.W., L.T., I.C., M.S., I.Z., A.L., M.B., P.L./ Design and execution of the biostatistical analysis: F.M., P.L., A.L./Interpretation of the statistical data: F.M., P.L., A.L./Drafting the manuscript: F.M., P.L./Revising the manuscript: F.M., S.R., V.D., C.W., L.T., I.C., M.S., I.Z., A.L., M.B., P.L. All authors read and approved the final manuscript.

\section{Competing interests}

The authors declare no competing interests.

\section{Additional information}

Supplementary information is available for this paper at https://doi.org/10.1038/s41598-020-65503-1.

Correspondence and requests for materials should be addressed to F.M.

Reprints and permissions information is available at www.nature.com/reprints.

Publisher's note Springer Nature remains neutral with regard to jurisdictional claims in published maps and institutional affiliations.

Open Access This article is licensed under a Creative Commons Attribution 4.0 International License, which permits use, sharing, adaptation, distribution and reproduction in any medium or format, as long as you give appropriate credit to the original author(s) and the source, provide a link to the Creative Commons license, and indicate if changes were made. The images or other third party material in this article are included in the article's Creative Commons license, unless indicated otherwise in a credit line to the material. If material is not included in the article's Creative Commons license and your intended use is not permitted by statutory regulation or exceeds the permitted use, you will need to obtain permission directly from the copyright holder. To view a copy of this license, visit http://creativecommons.org/licenses/by/4.0/.

(C) The Author(s) 2020 